\title{
Trends and projection of demographic indices of the Libyan population using a fifty-year census data 1954-2016 \\ Mohamed A Daw ${ }^{1}$ \& Abdallah HU El-Bouzedi ${ }^{2}$ \\ ${ }^{1}$ Department of Medical Microbiology, Faculty of Medicine, University of Tripoli, Tripoli, Libya, \\ ${ }^{2}$ Department of Laboratory Medicines, Faculty of Biotechnology, University of Tripoli, Tripoli, Libya, \\ Email: mohamedadaw@gmail.com
}

\begin{abstract}
Background: Libya is a North African country with the longest coast on the Mediterranean basin facing Europe. Little is known about the demographic characteristics of the Libyan population. Herein, we examine the demographic features and disparities in age and gender among Libyans over the last fifty years, which could be useful for future planning and allocation of resources.

Data and methods: Census data were used to analyze the demographic parameters and age trends from 1954 to 2016 by decades.

Findings The total Libyan population increased with significant sex differences during all decades. Fertility rates increased and mortality rates decreased. There had been more males during the first three decades but then their percentage declined since 1984, with women accounting for $49 \%$ in 2006 . The working-age population increased to $67 \%$ by 2006 .

Conclusion: These findings are discussed in the light of major demographic changes that has occurred in the Libyan society. Libya as a whole has enjoyed low mortality and great improvement in socioeconomic status.
\end{abstract}

Keywords: Libya, census data, demography, mortality, infertility, population

\section{Introduction}

The relationship between population characteristics and socio-economic development is well established. Demographic parameters such as population growth, structure and distribution have a great influence on economic status as well as on social and gender inequalities worldwide [Vogel and Porter, 2016; Wang etal, 1913]. Population census is the source of the basic population data required for planning and administration and also for many aspects of economic and social research [Coast etal. 2013; Groves, 20I0]. The major overriding criteria for deciding on the amount of information collected in censuses and surveys and from vital registration systems have been the planning needs and the availability of financial and human resources for carrying out the plans [Huselid 1995; Tejada etal, 2014].

The scope, nature and availability of demographic data vary throughout the world. Collecting population data by age and sex over time is difficult not only in developing countries but also in developed countries with established statistical offices and routinely organized censuses [Meyer etal, 2015; Hsu etal. 2014]. The United Nations (UN, 2005) projected that the percentage of the population over during the same period $[17, \mid 8]$. Hence evaluation of demographic and health parameters are needed for http://aps.journals.ac.za
60 years of age will rise from the $10 \%$ figure of 2000 to $20 \%$ by 2025 and to $31 \%$ by 2050 , while the percentage of the working-age population (I5-59 years) will fall by more than ten percentage points during the same period [ United Nation Report 1995]. It has been suggested that sometime between 2015 and 2020 the growth of the working age population will become negative, which in turn indicates that the growth in gross domestic product will suffer [Lutz etal., 2007; Shetty 20I2]. Many developing countries are currently undergoing rapid demographic and economic transitions, and particularly the proportion of aged individuals in lower- and middle-income nations [Hatton 201I; Andersen 1991]. In Africa, there is growing concern about the demographics in the coming decades due to the absence of an efficient registration system in most African countries [United Nation Report 2008]. However, many attempts were made in the beginning of the previous century to overcome this deficiency in both North and Sub-Saharan Africa [Booysen 2008; Anyangwe and Mtonga 2007]. The population growth rate in Africa estimated by united Nation (2.2\%) was higher than that of North America (1\%), Asia (1.3\%) and Europe (-0.1\%) future planning. Libya, the second largest country in Africa, with longest coast on the Mediterranean 
facing Europe and is one of the richest countries. The country has experienced considerable social, economic and political changes over the last 50 years. Studying these changes in Libya could be used as a model for North-African, Sub-Saharan and Arab countries [Daw etal. 2014; Daw etal. 2019 Daw etal. 2019]. National censuses are among the most useful tools for analyzing bio-data and demographic changes over time. Such surveys provide the practical indicators of the quality of life, social structure, and poverty. They are also the source of data for planning economic and health improvements [CAI 2013; Ross etal. 200]. In Libya, censuses have been conducted roughly every decade. Hence then we used these censuses to examine the demographic features and disparities in age and gender among Libyans over the last 50 years and how they could be used for planning and allocation of resources.

\section{Methods}

Birth and death statistics in Libya

Data for 1954-1967 were not available at a central location and were collected by personal contact from different resources. Before 1969, provinces was missing and monthly data were estimated. But since then, the Office of National Statistics (ONS) has published those statistics for all the provinces in Libya monthly. Moreover, since 1969, the ONS did not attempt to make any adjustments in the data on births as was done in the past; the births and deaths were reported by the municipalities without any adjustments. Since 1993, all the statistics on births and deaths have been reported monthly to the Authority for Information and Communication (GAIC).

\section{Data analysis}

To model the population growth rate from 1954 to 2006, the available census data were analyzed by the least squares method and by geometric growth rate. The geometric growth model has been adopted by the Libyan General Authority for Information and Communication and the data were collected from all over the Libyan regions and districts [Supplementary].

Supplementary 3 -Figure; Map of Libya showing the geographical locations and administrative boundaries of regions and districts involved in the study; Eastern Region districts [I-7], West Region Districts [8-16], South Regions Districts [17-22].

Supplementary-Table ; Libyan regions, Districts, administrative boundaries and population density

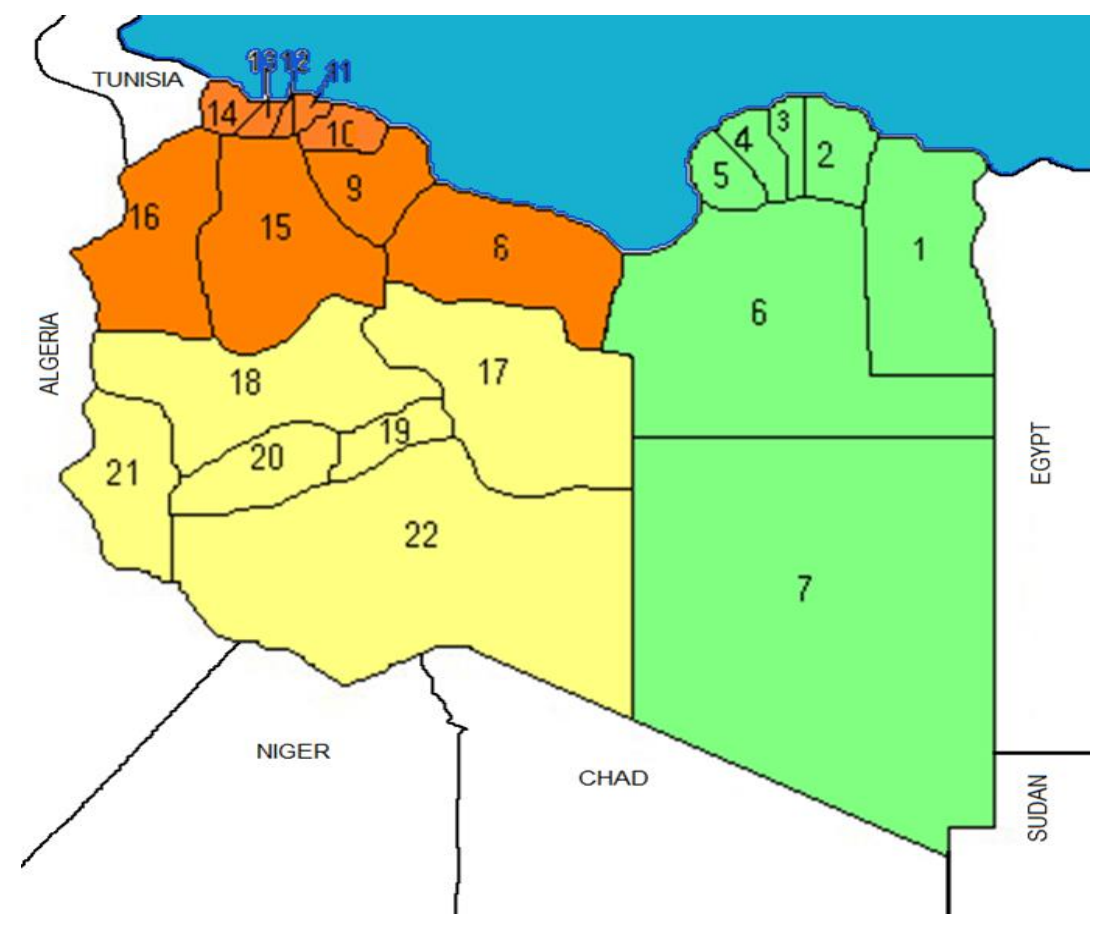


African Population Studies Vol 33, No. 2, 2019

\begin{tabular}{|c|c|c|c|c|}
\hline Arabic & English & Area $\left(\mathrm{km}^{2}\right)$ & Population & $\begin{array}{l}\text { Number } \\
\text { (on map) }\end{array}$ \\
\hline \multicolumn{5}{|c|}{ East Region[Cyrenaica] } \\
\hline البطنان & Butnan & 84,996 & 159,536 & I \\
\hline 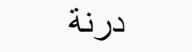 & Derna & 31,511 & $|63,35|$ & 2 \\
\hline الجبل الاخضر & ఏabal al Akhdar & 11,429 & 203,156 & 3 \\
\hline المرج & Marj & $13,5 \mid 5$ & 185,848 & 4 \\
\hline بنغازي & Benghazi & II,372 & 670,797 & 5 \\
\hline الواحات & Al Wahat & 105,523 & I77,047 & 6 \\
\hline الكفرة & Kufra & $433,6 \mathrm{II}$ & 50,104 & 7 \\
\hline \multicolumn{5}{|c|}{ West Region[Tripolitania] } \\
\hline سرت & $\underline{\text { Sirte }}$ & 225,437 & 193,720 & 8 \\
\hline 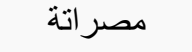 & Misrata & 29,172 & 550,938 & 9 \\
\hline 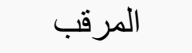 & Murqub & 6,796 & 432,202 & 10 \\
\hline طر ابلس & Tripoli & 835 & $1,065,405$ & 11 \\
\hline الجفارة & ఏafara & 2,666 & 453,198 & 12 \\
\hline الزاوية & Zawiya & 2,753 & 290,993 & 13 \\
\hline
\end{tabular}


African Population Studies Vol 33, No. 2, 2019

\begin{tabular}{|c|c|c|c|c|}
\hline Arabic & English & Area $\left(\mathrm{km}^{2}\right)$ & Population & $\begin{array}{l}\text { Number } \\
\text { (on map) }\end{array}$ \\
\hline \multicolumn{5}{|c|}{ East Region[Cyrenaica] } \\
\hline النقاط الخمس & Nuqat al Khams & 6,089 & 287,662 & 14 \\
\hline الجبل الغربي & Łabal al Gharbi & 76,717 & 304,159 & 15 \\
\hline 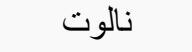 & Nalut & 67,191 & 93,224 & 16 \\
\hline \multicolumn{5}{|c|}{ South Region [Fezzan] } \\
\hline 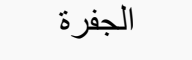 & Lufra & 117,410 & 52,342 & 17 \\
\hline و ادي الثـاطئ & $\underline{\text { Wadi al Shatii }}$ & 97,160 & 78,532 & 18 \\
\hline سبها & Sabha & 107,310 & 134,162 & 19 \\
\hline و ادي الحياة & Wadi al Hayaa & 31,485 & 76,858 & 20 \\
\hline 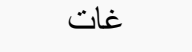 & Ghat & 68,482 & 23,518 & 21 \\
\hline 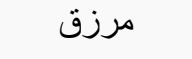 & Murzuq & 356,308 & 78,621 & 22 \\
\hline
\end{tabular}

\section{Results}

The total Libyan population in 1954 was I,041,599, had increased over five-fold to 5,323,991 and the of whom $51.9 \%$ were males. By 2006, the population percentage of males dropped to $50.6 \%$ (Tables I).

Table-I: The Libyan population censuses 1954-2006 as reported by the Libyan Office of National Statistics

\begin{tabular}{llll}
\hline Census year & Males & Females & Total \\
\hline 1954 & 540364 & 501235 & 1041599 \\
1964 & 788657 & 726844 & 1515501 \\
1973 & 1057919 & 994453 & 2052372
\end{tabular}


Table -2: The Libyan male population from 1954 to 2006.

\begin{tabular}{|c|c|c|c|c|c|c|c|c|c|c|}
\hline \multirow{2}{*}{ Year } & \multicolumn{9}{|c|}{ Age group (years) } & \multirow{2}{*}{ Total } \\
\hline & $0-9$ & $10-19$ & $20-29$ & $30-39$ & $40-49$ & $50-59$ & $60-69$ & 70-79 & $80+$ & \\
\hline 1954 & 146234 & 107567 & 91885 & 61551 & 43844 & 38083 & 27435 & 14259 & 9506 & 540364 \\
\hline 1955 & 50572 & 109266 & 93362 & 62906 & 44631 & 38302 & 27586 & $|44| 4$ & 9609 & 550648 \\
\hline 1956 & 59631 & 112732 & 96376 & 65703 & 46241 & 38749 & 27893 & 14725 & 9817 & 867 \\
\hline 1957 & 69187 & 116276 & 99457 & 68607 & 47895 & 39207 & 28206 & 15039 & 10026 & \\
\hline 1958 & 79266 & 119901 & 102607 & 71623 & 49594 & 39677 & 28526 & 15356 & 10237 & 787 \\
\hline 1959 & 189893 & 123606 & 105826 & 74753 & 51338 & 40160 & 28854 & 15674 & 10449 & 553 \\
\hline 1960 & 201097 & 127393 & 109114 & 78002 & 53129 & 40655 & 29188 & 15994 & 10663 & 235 \\
\hline 1961 & & 131 & 112473 & & 54966 & & & 16316 & & \\
\hline 1962 & 225 & 135216 & | I 5902 & 84873 & 56850 & 41686 & 29883 & 16640 & 11094 & 89 \\
\hline 1963 & 238450 & 139253 & 119403 & 88502 & 58784 & 42224 & 30242 & 16966 & $1|3| \mid$ & \\
\hline 1964 & 259446 & 145475 & 124795 & 94206 & 61778 & 43060 & 30800 & 17458 & 11639 & 657 \\
\hline 1965 & 266179 & 149290 & 125309 & 94775 & 62916 & 43415 & & 17546 & & \\
\hline 1966 & & & 12 & 95890 & 65236 & 23 & & 17717 & & \\
\hline 1967 & & 165 & 127245 & 96969 & 67607 & 44823 & & $|788|$ & 11920 & \\
\hline 1968 & 71 & 173987 & 128132 & 98010 & 70027 & 45514 & 31748 & 18036 & 12024 & \\
\hline 1969 & 325342 & 182907 & 128964 & 99010 & 72495 & 46195 & 31985 & 18182 & & \\
\hline 1970 & & 19 & 12 & 99967 & 75 & & & 18320 & & \\
\hline 1971 & & 20 & & $10088 \mid$ & 77572 & 47525 & & 18449 & 12299 & \\
\hline 1972 & 35 & 21 & 13 & 101750 & 80177 & 48172 & 00 & 18569 & 12379 & 636 \\
\hline 1973 & 401 & 226414 & 131295 & 102431 & 83732 & 48863 & 32680 & 18633 & 12422 & \\
\hline 1974 & & & & & & & & & & \\
\hline 1975 & & & & & & & & 19325 & & \\
\hline 1976 & & 262 & 15 & 108212 & 88149 & 55 & & 19795 & 13197 & 649 \\
\hline 1977 & & 278 & & 110579 & 89942 & & & 20272 & 13515 & 606 \\
\hline 1978 & 465669 & 295886 & 167295 & I I 2976 & 91748 & 60524 & & 20755 & & 529 \\
\hline 1979 & & & & 02 & & & & 21244 & & \\
\hline 1980 & 49 & 332 & 18 & 117856 & 95396 & 66488 & 02 & 21740 & 14493 & 598 \\
\hline 1981 & & & 19 & 120339 & 97237 & 69667 & & $2224 I$ & 14827 & 916 \\
\hline 1982 & 529319 & 374138 & $20707 \mid$ & 122849 & 99088 & 72985 & 181 & 22748 & & 544 \\
\hline 1983 & & & & & & & & 23261 & & \\
\hline 1984 & & & 23 & 66 & 13 & 45 & 63 & 24086 & & \\
\hline 1985 & 57 & $44 \mid 276$ & 244772 & 133408 & 105089 & 90 & 06 & 24381 & 16254 & 058 \\
\hline 1986 & $5760 \mid 4$ & 454629 & 260102 & 141629 & 107447 & 85701 & 52952 & 24977 & $1665 \mid$ & 0102 \\
\hline 1987 & & & 276254 & 150288 & 109799 & & & 25578 & & \\
\hline 1988 & & & & & & & & 26183 & & \\
\hline 1989 & $57 \varepsilon$ & 495944 & 31 & 168991 & I 14477 & 92725 & 17 & 26792 & $|786|$ & 971 \\
\hline 1990 & 578327 & 510111 & 329972 & |7907| & 116796 & 95089 & 62252 & 27404 & 18270 & 7292 \\
\hline 1991 & 578136 & 524462 & 349746 & 189662 & 119098 & 97459 & 64763 & 28020 & & 0026 \\
\hline 1992 & & & & & & & & 28637 & & \\
\hline 1993 & 576819 & 553683 & 392306 & 212455 & 123635 & 102210 & 70014 & 29257 & 19505 & 2079884 \\
\hline 1994 & 575692 & 568536 & 415164 & 224695 & 125865 & 104584 & 72757 & 29878 & 19919 & 2137090 \\
\hline 1995 & 573266 & 592396 & 453697 & 245331 & 129338 & 108339 & 77270 & 30865 & 20577 & 2231079 \\
\hline 1996 & 578354 & 597654 & 457724 & 247508 & 130486 & 109301 & 77956 & 31139 & 20759 & 2250881 \\
\hline 1997 & 588666 & 608310 & 465885 & 251922 & 132813 & 111249 & 79346 & 31694 & 21130 & 2291015 \\
\hline 1998 & 599192 & 619187 & 474215 & 256426 & 135187 & 113239 & 80764 & 32261 & 21507 & 2331978 \\
\hline
\end{tabular}




\begin{tabular}{|c|c|c|c|c|c|c|c|c|c|c|}
\hline 1999 & 609905 & 630258 & 482694 & 261011 & 137604 & 115263 & 82208 & 32838 & 21892 & 2373673 \\
\hline 2000 & 620840 & 641558 & 491348 & 265690 & $|4007|$ & 117330 & 83682 & 33427 & 22284 & 2416230 \\
\hline 2001 & 631910 & 652997 & 500109 & 270428 & 142569 & 119422 & 85174 & 34023 & 22682 & 2459314 \\
\hline 2002 & 643208 & 664672 & 509051 & 275263 & 145118 & 121557 & 86697 & 34631 & 23087 & 2503284 \\
\hline 2003 & 654708 & 676556 & 518153 & 280184 & $1477 \mid 2$ & 123731 & 88247 & 35250 & 23500 & 2548041 \\
\hline 2004 & 666447 & 688686 & 527443 & 285208 & $|5036|$ & 125949 & 89830 & 35882 & 23921 & 2593727 \\
\hline 2005 & 678330 & 700966 & 536847 & 290293 & 153042 & 128195 & 91431 & 36522 & 24348 & 2639974 \\
\hline 2006 & 560982 & 576790 & 588420 & 445562 & 226353 & 120372 & 99430 & 46342 & 30894 & $2695 \mid 45$ \\
\hline
\end{tabular}

Table 3: The Libyan female population from 1954 to 2006.

\begin{tabular}{|c|c|c|c|c|c|c|c|c|c|c|}
\hline \multirow{2}{*}{ Year } & \multicolumn{9}{|c|}{ Age groups (years) } & \multirow{2}{*}{ Total } \\
\hline & $0-9$ & $10-19$ & $20-29$ & $30-39$ & $40-49$ & $50-59$ & $60-69$ & 70-79 & $80+$ & \\
\hline 1954 & 144994 & 92215 & 82591 & 58197 & 43569 & 31845 & 23958 & 14320 & 9546 & 501235 \\
\hline 1955 & 149139 & 93938 & 84174 & 59356 & 44187 & 32003 & 23948 & 14319 & 9546 & 510610 \\
\hline 1956 & 157762 & 97458 & 87411 & 61733 & 45440 & 32340 & 23942 & 14314 & 9542 & 529943 \\
\hline 1957 & 166813 & 101069 & 90734 & 64179 & $467 \mid 4$ & 32705 & 23957 & 14302 & 9535 & 550008 \\
\hline 1958 & 176312 & I0477| & 94145 & 66696 & 48009 & 33101 & 23993 & 14284 & 9523 & 570833 \\
\hline 1959 & 186277 & 108565 & 97644 & 69286 & 49324 & 33530 & 24053 & $|426|$ & 9507 & 592447 \\
\hline 1960 & 196730 & 112453 & 101233 & 71951 & 50661 & 33995 & 24136 & 14232 & 9488 & \\
\hline$|96|$ & 207692 & 116437 & 104915 & 74692 & 52019 & 34498 & 24245 & 14197 & 9465 & 638159 \\
\hline 1962 & 219185 & 120519 & 108689 & 77511 & 53399 & 35042 & 24380 & 14158 & 9438 & 662322 \\
\hline 1963 & 231233 & 124700 & 112560 & 80411 & 54802 & 35631 & 24543 & 14113 & 9409 & 687399 \\
\hline 1964 & 250415 & 131167 & 118553 & 84918 & 56951 & 36603 & 24842 & 14037 & 9358 & 726844 \\
\hline 1965 & 256 & 13 & 119023 & 85421 & 57997 & 36914 & 60 & 09 & 06 & 28 \\
\hline 1966 & 270348 & 141663 & 119929 & 86407 & 60129 & 37533 & 25188 & 14247 & 9498 & 4943 \\
\hline 1967 & 285021 & |4943| & $|2| 24 \mid$ & 88651 & 61367 & 38319 & 26219 & 14918 & 9946 & 795112 \\
\hline 1968 & 298882 & $|567| \mid$ & 121590 & 88277 & 64530 & 38753 & 25609 & 14506 & 9671 & 818528 \\
\hline 1969 & 314004 & 164696 & 122342 & 89158 & 66797 & 39 & 25802 & 14625 & 9750 & 525 \\
\hline 1970 & 477 & 174177 & 124065 & 93086 & 66859 & 40329 & 27870 & $|598|$ & 10654 & 498 \\
\hline 1971 & 346013 & 181626 & 123679 & 90803 & 71458 & 40523 & 26148 & 14843 & 9895 & 986 \\
\hline 1972 & 362920 & 190582 & 124262 & 91564 & 73850 & 41093 & 26301 & 14940 & 9960 & 935471 \\
\hline 1973 & 390867 & $20624 I$ & 126090 & 97640 & 73104 & & & 17124 & 11416 & 994453 \\
\hline 1974 & 39 & & 83 & 98845 & 73998 & & & & 11589 & 367 \\
\hline 1975 & 410566 & 227335 & 136245 & 101295 & 75808 & 45935 & 31607 & 17912 & 11941 & 8643 \\
\hline 1976 & 424120 & 242526 & 143466 & 103786 & 77638 & 48480 & 32998 & $|845|$ & 12301 & 1103765 \\
\hline 1977 & 438004 & 258670 & 151063 & 106319 & 79490 & 51154 & $3444 I$ & 19001 & 12667 & 1150809 \\
\hline 1978 & 452221 & 275823 & 159055 & 108894 & 81360 & 53964 & 39 & 19561 & I304| & \\
\hline 1979 & 466773 & 294041 & 167463 & $11|5| \mid$ & 83250 & 56916 & 37493 & 20132 & $1342 \mid$ & 0999 \\
\hline 1980 & $48|66|$ & 313386 & 176305 & 114169 & 85158 & 60015 & 39104 & $207 / 3$ & 13808 & 1304319 \\
\hline 1981 & 496887 & 333921 & 185604 & 116869 & 87082 & 63269 & 40774 & 21304 & 14203 & |3599|| \\
\hline 1982 & & 355713 & 195383 & 119609 & 89022 & 66683 & 42504 & & 14604 & 1417873 \\
\hline 1983 & & 37 & 205663 & 122389 & 90977 & & 44297 & & 150|| & 3306 \\
\hline 1984 & 554278 & 418408 & 223063 & $12688 \mid$ & 94106 & 76311 & 47272 & 23507 & $|567|$ & 1579497 \\
\hline 1985 & 555181 & 424935 & 230401 & 130677 & 95645 & 77158 & 48153 & 23819 & 15879 & 1601848 \\
\hline 1986 & 556767 & 438193 & 245759 & 138586 & 98768 & 78854 & 49960 & 24447 & 16298 & 1647632 \\
\hline 1987 & 558047 & 451669 & 262006 & 146905 & 101936 & 80543 & 51822 & 25078 & 16718 & 1694723 \\
\hline 1988 & 559016 & 465358 & 279183 & 155647 & 105147 & 82222 & 53739 & 25710 & 17140 & $|743| 6 \mid$ \\
\hline 1989 & 559669 & 479250 & 297331 & 164829 & 108398 & 83890 & $557 / 3$ & 26342 & $1756 \mid$ & 1792984 \\
\hline 1990 & 560003 & 493337 & 316492 & 174467 & 111686 & 85543 & 57744 & 26974 & 17983 & 1844230 \\
\hline 1991 & 560015 & 507611 & 336709 & 184577 & 115007 & 87180 & 59834 & 27606 & 18404 & $|89694|$ \\
\hline 1992 & 559702 & 522060 & 358025 & 195175 & 118359 & 88797 & 61982 & 28235 & 18823 & 1951158 \\
\hline 1993 & 559063 & 536676 & 380486 & 206277 & 121738 & 90392 & 64191 & $2886 \mid$ & $1924 \mid$ & 2006925 \\
\hline 1994 & 558098 & 551449 & 404138 & 217900 & 125140 & 91962 & 66460 & 29483 & 19656 & 2064286 \\
\hline 1995 & 555898 & 575175 & 444213 & 237446 & 130576 & 94397 & 70187 & 30461 & 20307 & 2158660 \\
\hline
\end{tabular}


African Population Studies Vol 33, No. 2, 2019

\begin{tabular}{|c|c|c|c|c|c|c|c|c|c|c|}
\hline 1996 & 561044 & 580500 & 448325 & 239644 & 131785 & 95271 & 70837 & 30743 & 20495 & 2178644 \\
\hline 1997 & $57 \mid 480$ & 591297 & 456665 & 244102 & 134236 & 97043 & 72154 & 31315 & 20876 & 2219168 \\
\hline 1998 & 603804 & 648416 & 496601 & 268531 & 141569 & 118584 & 84577 & 33784 & 22523 & 2418388 \\
\hline 1999 & 592998 & 613561 & 473859 & 253293 & |3929| & 100697 & $7487 \mid$ & 32494 & 21663 & 2302725 \\
\hline 2000 & 604089 & 625037 & 482722 & 258030 & 141896 & 102580 & $7627 \mid$ & 33102 & 22068 & 2345795 \\
\hline 2001 & 615325 & 636663 & 491701 & 262830 & 144535 & 104488 & 77690 & 33717 & 22478 & 2389429 \\
\hline 2002 & 626803 & 648538 & 500872 & 267732 & $|4723|$ & 106437 & 79139 & 34346 & 22897 & 2433997 \\
\hline 2003 & 638494 & 660635 & 510215 & 272726 & 149978 & 108423 & 80615 & 34987 & 23325 & 2479396 \\
\hline 2004 & 638526 & 660669 & 510241 & 272740 & 149985 & 108428 & 80619 & 34989 & 23326 & 2479522 \\
\hline 2005 & 650403 & 672958 & 519732 & $2778 \mid 3$ & 152775 & 110445 & 82119 & 35639 & 23760 & 2525643 \\
\hline
\end{tabular}

The temporal trends of the estimated and observed census (Figure I) show a linear trend. Comparison of the outcomes of the linear and geometric models of the Libyan population from 1954 to 2006 indicates linear population growth (Figure 2).
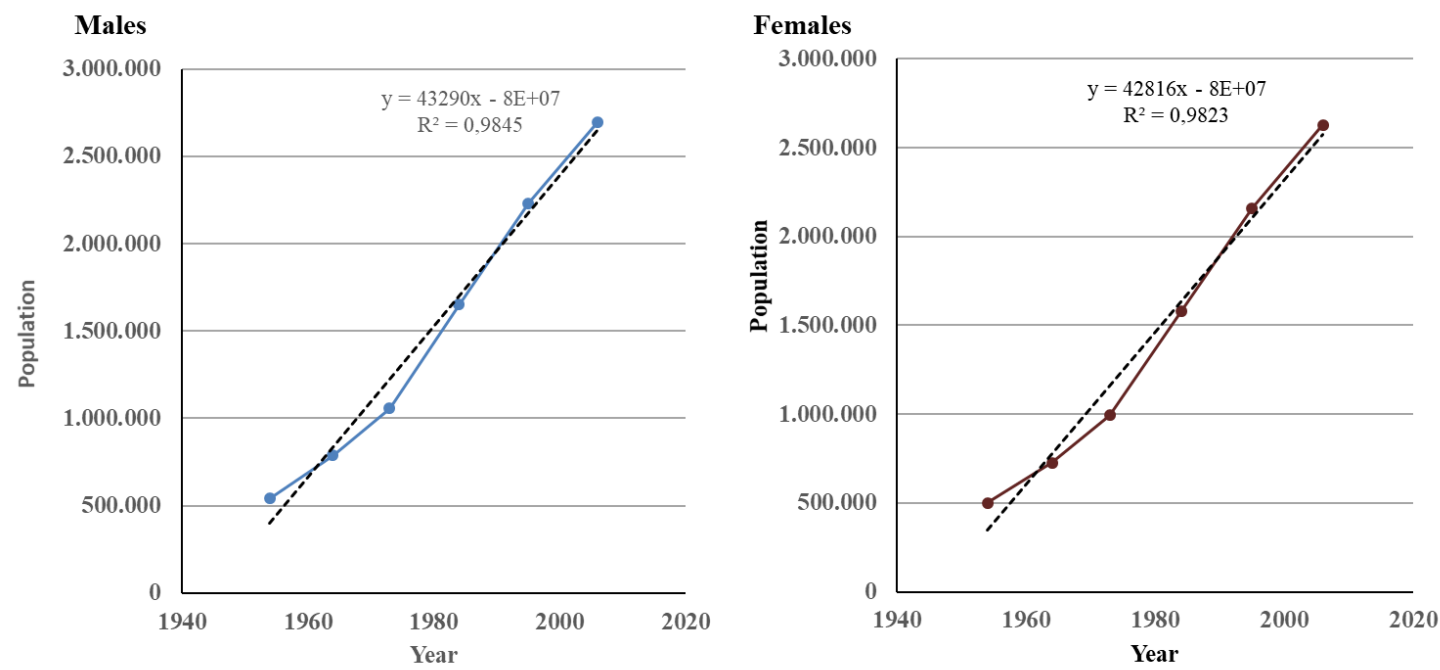

Figure I: The Libyan populations' census as reported by the Libyan Office of National Statistics. The dotted line shows the linear regression line.
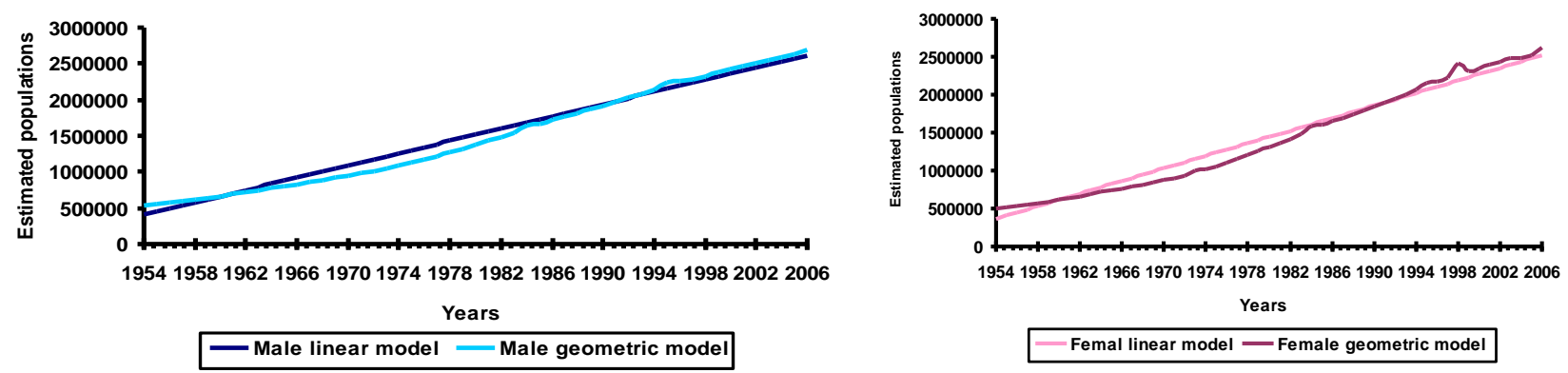

Figure 2: Linear and geometric modeling of Libyan population growth during 1954-2006 based on the national censuses.

The trends of fertility and mortality rates between 1954 and 2006 in the Libyan population were analyzed. The number of births increased until 1992 but plunged precipitously in the following year (Figure 3). 


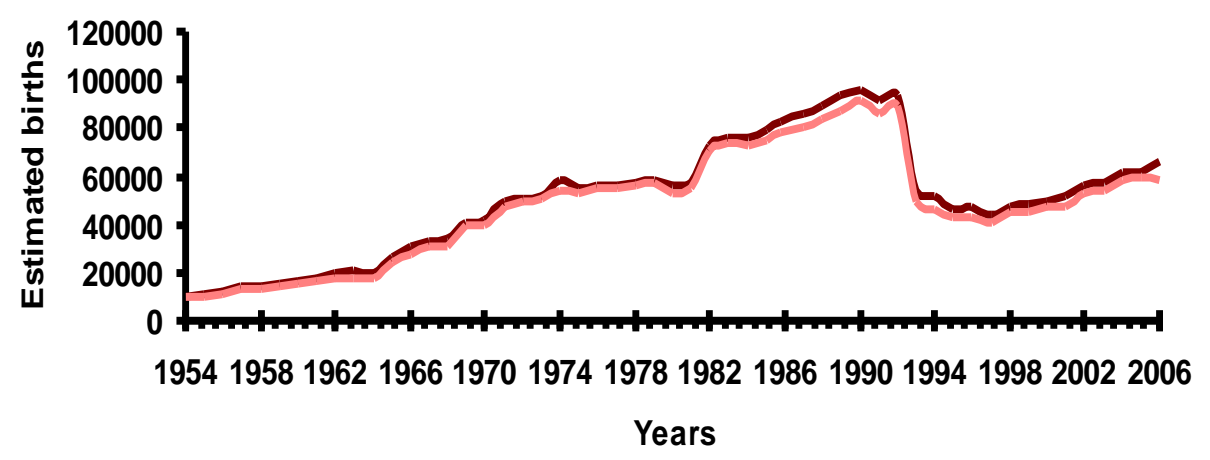

\section{- Male - Fimale}

Figure 3: Estimated Libyan fertility 1954-2006

The same was true for the estimated annual number of deaths (Figure 4). Figure 5

shows the age-gender profile of the Libyan population, during2016.

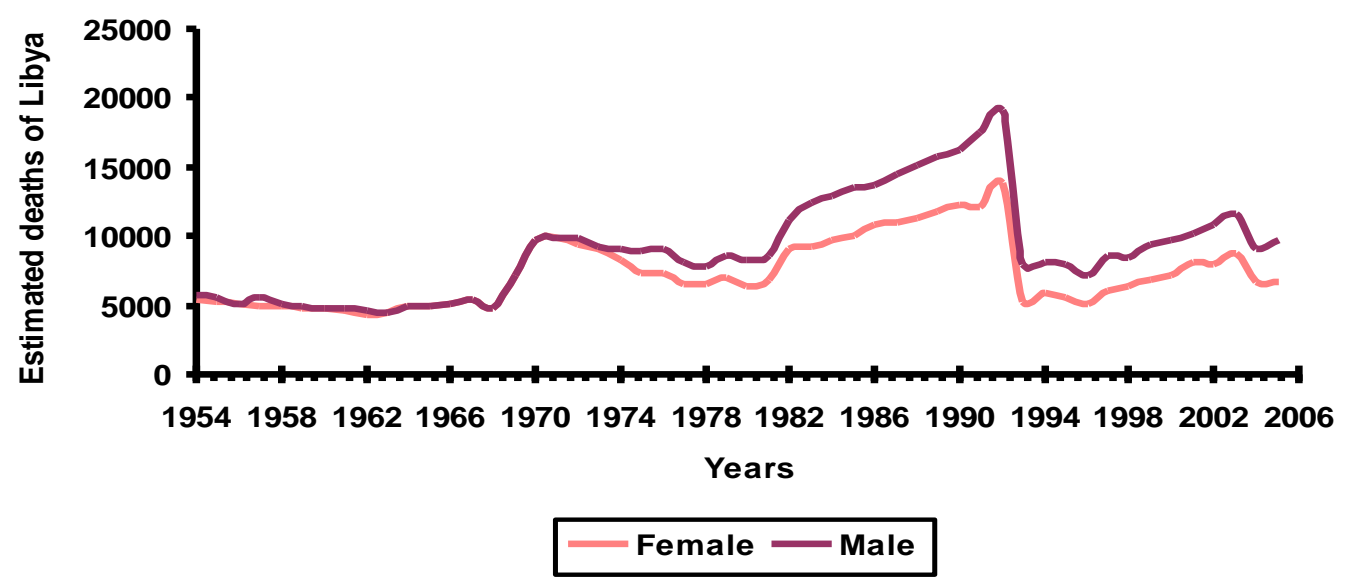

Figure 4: Estimated Libyan mortality 1954-2006 


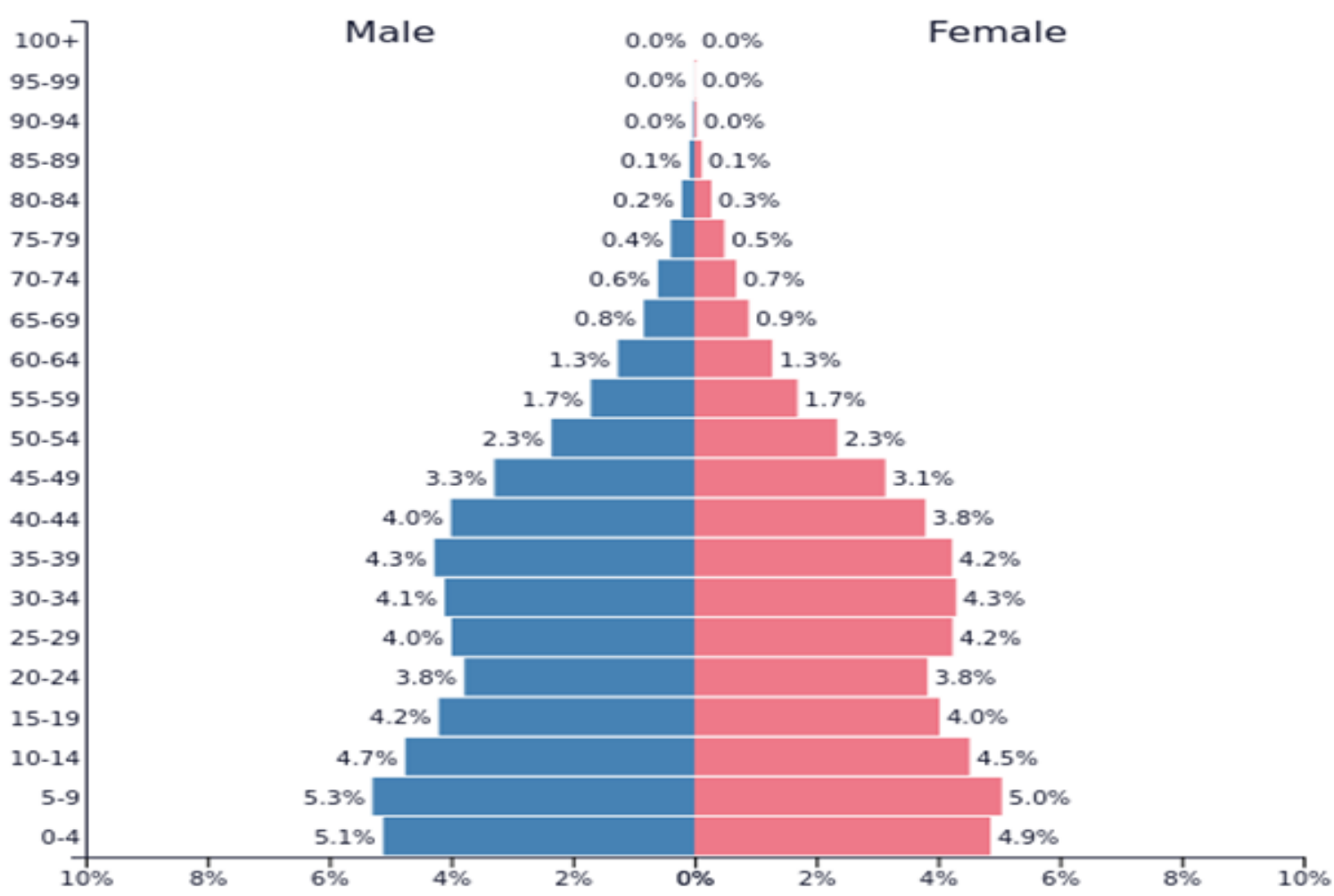

Figure 5; Age-gender profile of the Libyan population in person-years. (M;F) 2016

This anomaly was attributed to the adjustments and estimates made by the National Statistics Office before 1993. But upon establishment of the General Authority for Information and Communication (GAIC) in 1993, the vital statistics data started to be sent directly from civil record offices to GAIC without making any estimates.

The number of deaths in the Libyan population from all causes from 1954 to 2006 is shown in table 4 (males) and table 5 (females).

Table-4: Male mortality from all causes.

\begin{tabular}{|c|c|c|c|c|c|c|c|c|c|}
\hline \multirow{2}{*}{ Year } & \multicolumn{9}{|c|}{ Age group (years) } \\
\hline & $0-9$ & $10-19$ & $20-29$ & $30-39$ & $40-49$ & $50-59$ & $60-69$ & $70-79$ & $80+$ \\
\hline 1954 & 2733 & 311 & 245 & 188 & 345 & 211 & 423 & 578 & 644 \\
\hline 1955 & 2677 & 356 & 268 & 209 & 278 & 215 & 465 & 524 & 593 \\
\hline 1956 & 2493 & 312 & 256 & 178 & 264 & 233 & 367 & 489 & 573 \\
\hline 1957 & 2517 & 316 & 256 & 293 & 234 & 273 & 445 & 562 & 614 \\
\hline 1958 & 2381 & 267 & 233 & 261 & 222 & 233 & 423 & 488 & 576 \\
\hline 1959 & 2346 & 251 & 229 & 244 & 223 & 244 & 442 & 479 & 568 \\
\hline 1960 & 2305 & 254 & 246 & 232 & 241 & 261 & 455 & 471 & 552 \\
\hline 1961 & 2076 & 298 & 266 & 199 & 233 & 245 & 467 & 497 & 566 \\
\hline 1962 & 1948 & 166 & 237 & 259 & 188 & 235 & 478 & 445 & 678 \\
\hline 1963 & 1944 & 197 & 211 & 188 & 211 & 215 & 534 & 366 & 589 \\
\hline 1964 & 2169 & 246 & 178 & 213 & 189 & 246 & 673 & 389 & 564 \\
\hline 1965 & 2133 & 269 & 263 & 195 & 156 & 256 & 567 & 495 & 621 \\
\hline 1966 & 2440 & 278 & 178 & 158 & 162 & 216 & 621 & 567 & 533 \\
\hline 1967 & 2276 & 184 & 256 & 197 & 178 & 358 & 765 & 480 & 657 \\
\hline 1968 & 2236 & 167 & 189 & 157 & 143 & 240 & 528 & 562 & 580 \\
\hline 1969 & 3677 & 245 & 287 & 232 & 254 & 362 & 684 & 752 & 732 \\
\hline 1970 & 5466 & 287 & 305 & 284 & 367 & 432 & 745 & 866 & 896 \\
\hline 1971 & 6558 & 317 & 324 & 292 & 401 & 453 & 782 & 923 & 1021 \\
\hline
\end{tabular}


African Population Studies Vol 33, No. 2, 2019

\begin{tabular}{|c|c|c|c|c|c|c|c|c|c|}
\hline 1972 & 6268 & 286 & 292 & 269 & 361 & 409 & 704 & 833 & 920 \\
\hline 1973 & 5578 & 255 & 260 & 239 & 321 & 365 & 626 & $74 I$ & 819 \\
\hline 1974 & 5483 & 250 & 255 & 235 & 316 & 358 & 616 & 729 & 805 \\
\hline 1975 & 3795 & 368 & 410 & 326 & 403 & 527 & 700 & 862 & 1500 \\
\hline 1976 & 3836 & 435 & 456 & 313 & 376 & 553 & 802 & 1045 & 1217 \\
\hline \begin{tabular}{|l}
1977 \\
\end{tabular} & 3509 & 334 & 342 & 255 & $34 I$ & 469 & 649 & 812 & 1422 \\
\hline \begin{tabular}{|l|}
1978 \\
\end{tabular} & 3355 & 319 & 327 & 244 & 326 & 448 & 621 & 776 & 1360 \\
\hline 1979 & 3694 & 358 & 399 & 317 & 392 & 513 & 681 & 839 & 1460 \\
\hline 1980 & 3545 & 402 & 421 & 289 & 347 & 511 & $74 I$ & 966 & 1125 \\
\hline 1981 & 3643 & 413 & 433 & 297 & 357 & 525 & 762 & 993 & 1156 \\
\hline 1982 & $448 I$ & 501 & 468 & 338 & 464 & 733 & 980 & 1427 & 1713 \\
\hline 1983 & 5163 & 568 & 571 & 340 & 509 & 878 & 1060 & 1519 & 1862 \\
\hline 1984 & 5070 & 662 & 623 & 439 & 484 & 884 & 1256 & 1425 & 2047 \\
\hline 1985 & 4993 & 622 & 580 & 386 & 519 & 1040 & 1299 & 1563 & 2508 \\
\hline 1986 & 4863 & 645 & 695 & 370 & 487 & 1037 & 1457 & 1734 & 2332 \\
\hline 1987 & 4884 & 711 & 823 & 483 & 621 & 1163 & 1593 & $165 \mid$ & 2611 \\
\hline 1988 & 4822 & 750 & 793 & 496 & 619 & 11164 & 1763 & 2053 & 2650 \\
\hline 1989 & 4717 & 751 & 878 & 724 & 683 & 1082 & 1912 & 2009 & 2942 \\
\hline 1990 & 5065 & 774 & 845 & 596 & 715 & $|24|$ & 1865 & 2149 & 2920 \\
\hline 1991 & 4728 & 931 & $|23|$ & 723 & 782 & 1334 & 2428 & $227 \mid$ & 3046 \\
\hline 1992 & 5162 & 860 & $|139|$ & 1208 & 911 & 1435 & 2552 & 2532 & 3075 \\
\hline 1993 & 1899 & 392 & 649 & 385 & 397 & 693 & 1158 & 11112 & 1267 \\
\hline 1994 & 2218 & 370 & 566 & 473 & 368 & 595 & 1108 & 1107 & 1362 \\
\hline 1995 & 1357 & 319 & 600 & 674 & 559 & 657 & 1076 & 1249 & 1406 \\
\hline 1996 & $|26|$ & 339 & 539 & 503 & 392 & 618 & 1122 & 1222 & 1185 \\
\hline \begin{tabular}{|l|}
1997 \\
\end{tabular} & 1459 & 360 & 597 & 581 & 470 & 705 & 1458 & 1580 & 1333 \\
\hline 1998 & 1317 & 359 & 529 & 425 & 444 & 766 & 1499 & 1695 & $|44|$ \\
\hline 1999 & 1455 & 394 & 596 & 507 & 501 & 820 & 1594 & 1960 & 1632 \\
\hline 2000 & 1616 & 386 & 687 & 562 & 548 & 844 & 1599 & 1982 & 1515 \\
\hline 2001 & 1844 & 393 & 660 & 603 & 586 & 790 & 1707 & 1999 & 1577 \\
\hline 2002 & 1811 & 418 & 732 & 732 & 570 & 813 & 1726 & 2233 & 1828 \\
\hline 2003 & 1746 & 433 & 809 & 800 & 664 & 872 & 1875 & 2359 & 2056 \\
\hline 2004 & 1533 & 338 & 562 & 644 & 552 & 683 & 1326 & 1783 & 1731 \\
\hline 2005 & 1780 & 297 & 498 & 665 & 718 & 704 & 1424 & 1857 & 1727 \\
\hline 2006 & 1308 & 286 & 602 & 765 & 772 & 842 & 1671 & 2229 & 1842 \\
\hline
\end{tabular}

Table -5: Female mortality from all causes

\begin{tabular}{|c|c|c|c|c|c|c|c|c|c|}
\hline \multirow{2}{*}{ Year } & \multicolumn{10}{|c|}{ Age group (years) } \\
\cline { 2 - 10 } & $\mathbf{0 - 9}$ & $10-19$ & $\mathbf{2 0 - 2 9}$ & $30-39$ & $\mathbf{4 0 - 4 9}$ & $\mathbf{5 0 - 5 9}$ & $\mathbf{6 0 - 6 9}$ & $\mathbf{7 0 - 7 9}$ & $\mathbf{8 0 +}$ \\
\hline 1954 & 2533 & 311 & 245 & 188 & 345 & 211 & 423 & 578 & 544 \\
\hline 1955 & 2477 & 356 & 268 & 209 & 278 & 215 & 385 & 474 & 593 \\
\hline 1956 & 2493 & 312 & 256 & 178 & 264 & 233 & 367 & 489 & 573 \\
\hline 1957 & 2217 & 316 & 256 & 293 & 234 & 273 & 445 & 462 & 514 \\
\hline 1958 & 2281 & 267 & 233 & $26 I$ & 222 & 233 & 423 & 488 & 476 \\
\hline 1959 & 2105 & 254 & 246 & 232 & 241 & $26 I$ & 455 & 471 & 452 \\
\hline 1960 & 2005 & 234 & 226 & 212 & 221 & 251 & 435 & $45 I$ & 462 \\
\hline 1961 & 1918 & 228 & 216 & 199 & 233 & 245 & 467 & 497 & 466 \\
\hline
\end{tabular}


African Population Studies Vol 33, No. 2, 2019

\begin{tabular}{|c|c|c|c|c|c|c|c|c|c|}
\hline 1962 & 1848 & 166 & 237 & 259 & 188 & 235 & 378 & 445 & 578 \\
\hline 1963 & 1944 & 197 & 211 & 188 & 211 & 215 & 534 & 366 & 589 \\
\hline 1964 & 2169 & 246 & 178 & 213 & 189 & 246 & 673 & 389 & 564 \\
\hline 1965 & 2133 & 269 & 263 & 195 & 156 & 256 & 567 & 495 & 621 \\
\hline 1966 & 2440 & 278 & 178 & 158 & 162 & 216 & 621 & 567 & 533 \\
\hline 1967 & 2276 & 184 & 256 & 197 & 178 & 358 & 765 & 480 & 657 \\
\hline 1968 & 2236 & 167 & 189 & 157 & 143 & 240 & 528 & 562 & 580 \\
\hline 1969 & 3677 & 245 & 287 & 232 & 254 & 362 & 684 & 752 & 732 \\
\hline 1970 & 5466 & 287 & 305 & 284 & 367 & 432 & 745 & 866 & 896 \\
\hline |97| & 5558 & 317 & 324 & 292 & 401 & 453 & 782 & 923 & 921 \\
\hline 1972 & 6055 & 257 & 223 & 257 & 227 & 262 & 549 & 694 & 897 \\
\hline 1973 & 5808 & 246 & 214 & 247 & 218 & 251 & 527 & 666 & 861 \\
\hline 1974 & 5280 & 224 & 194 & 224 & 198 & 228 & 479 & 605 & 782 \\
\hline 1975 & 3396 & 247 & 221 & 208 & 268 & 337 & 544 & 736 & 1300 \\
\hline 1976 & 3656 & 262 & 144 & 207 & 237 & 349 & 599 & 814 & 1018 \\
\hline 1977 & 3056 & 223 & 199 & 187 & 242 & 304 & 491 & 664 & 1178 \\
\hline 1978 & 3102 & 219 & 175 & 183 & 240 & 298 & 488 & 673 & 1172 \\
\hline 1979 & 3254 & 237 & 212 & 199 & 257 & 323 & 522 & 706 & 1246 \\
\hline 1980 & 3099 & 222 & 122 & 175 & 201 & 296 & 507 & 689 & 1029 \\
\hline 1981 & 3437 & 246 & 135 & 194 & 223 & 328 & 563 & 765 & 957 \\
\hline 1982 & 4330 & 291 & 227 & 276 & 346 & 468 & 748 & 1053 & $|39|$ \\
\hline 1983 & 4275 & 283 & 262 & 257 & 288 & 489 & 798 & 1130 & 1518 \\
\hline 1984 & 4358 & 324 & 262 & 237 & 320 & 507 & 778 & 1200 & 1784 \\
\hline 1985 & 4270 & 301 & 270 & 255 & 332 & 574 & 870 & 1282 & 1876 \\
\hline 1986 & 4564 & 337 & 272 & 266 & 362 & 601 & 1014 & 1415 & 2009 \\
\hline 1987 & 4282 & 326 & 335 & 299 & 299 & 735 & 1097 & 1492 & 2124 \\
\hline 1988 & 4006 & 403 & 373 & 317 & 437 & 791 & 1127 & 1599 & 2247 \\
\hline 1989 & 3945 & 378 & 404 & 417 & 468 & 769 & 1232 & 1668 & 2461 \\
\hline 1990 & 4296 & 428 & 422 & 353 & 524 & 793 & 1211 & 1745 & 2568 \\
\hline 1991 & 3909 & 461 & 487 & 416 & 443 & 860 & $14 \mid 4$ & 1772 & 2385 \\
\hline 1992 & 4379 & 465 & 467 & 524 & 623 & 850 & 1649 & 1993 & 2901 \\
\hline 1993 & 1534 & 200 & 224 & 239 & 247 & 352 & 622 & $78 I$ & 1090 \\
\hline 1994 & 1852 & 198 & 198 & 217 & 255 & 361 & 700 & 853 & 1235 \\
\hline 1995 & 1076 & 182 & 224 & 460 & 358 & 392 & 702 & 902 & 1345 \\
\hline 1996 & 930 & 177 & 201 & 374 & 297 & 434 & 744 & 850 & 1093 \\
\hline 1997 & 1172 & 193 & 289 & 297 & 359 & 485 & 902 & 1127 & 1293 \\
\hline 1998 & 1125 & 192 & 253 & 337 & 397 & 510 & 1043 & 1218 & 1345 \\
\hline 1999 & 1190 & 193 & 268 & 349 & 407 & 541 & 1111 & 1304 & $|47|$ \\
\hline 2000 & 1285 & 243 & 224 & 324 & 472 & 716 & 1110 & 1339 & 1413 \\
\hline 2001 & 1582 & 270 & 300 & 386 & 479 & 682 & 1233 & 1600 & 1643 \\
\hline 2002 & 1449 & 230 & 260 & 442 & 480 & 599 & 1164 & I54I & 1742 \\
\hline 2003 & 1536 & 267 & 300 & 464 & 521 & 657 & 1252 & 1842 & 1965 \\
\hline 2004 & 1240 & 142 & 238 & 324 & 357 & 502 & 898 & 1256 & 1656 \\
\hline 2005 & 1395 & 174 & 191 & 328 & 399 & 426 & 890 & 1375 & 1577 \\
\hline 2006 & 1219 & 161 & 260 & 345 & 462 & 532 & 1216 & 1687 & 1776 \\
\hline
\end{tabular}

Figure 6 shows the number of deaths in males and females per age group, as well as the death rate per age group, in 2006. These rates are different http://aps.journals.ac.za between males and females, likely due to differences in lifestyle. The death rate from all causes ranged from $0-0.5 \%$ in age group $0-45$ years to $0.5-2 \%$ in 
age group $45-65$ years, and up to $6 \%$ above 65 years. The death rate was lower among those aged less

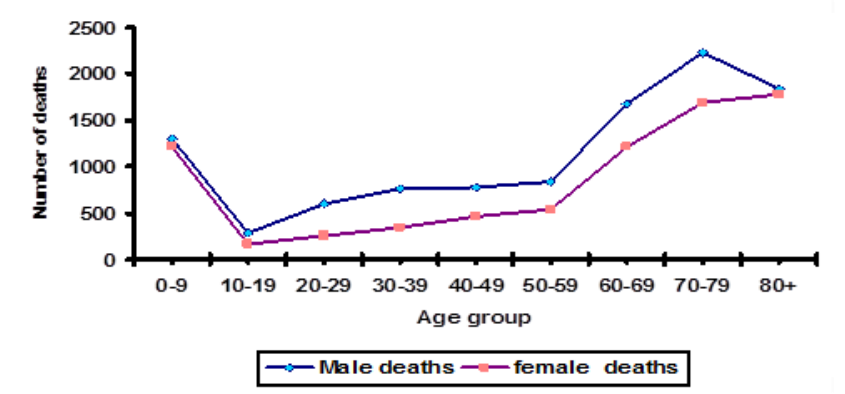

than 40 years. A difference in death rate between males and females emerged after the age of 40 years.

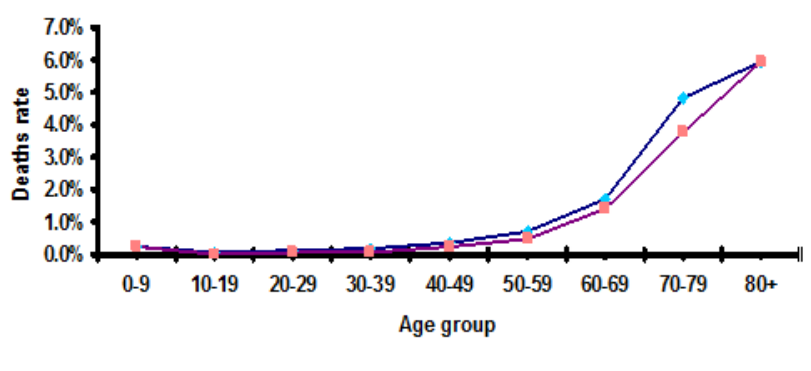

Figure 6: Libyan population deaths and death rates from all causes per age group in 2006.

\section{Discussion}

Population censuses provide the demographics of a whole population and therefore avoid issues of representativeness that often hamper estimates based on medical statistics. Recently, there has been extensive research in the area of census biodata using statistical models to provide a better understanding of the demographic parameters and to plan for future prospects [Byars-Winston etal. 2015]. In contrast to demographic surveys with limited sample sizes, the census is based on large numbers and provides narrow confidence intervals. The geometric and linear models are the most useful models for estimating the rate of growth during the intercensuses years and for extrapolating the findings to the future [Ribeiro and Petyt 199; Faraway 2014].

In this study, we combined these two statistical models to determine the key demographic parameters within the Libyan population during 1954-2006. These parameters include birth rates, sex ratios at birth, and age- and gender-specific death and life expectancies. Both males and females had linear growth patterns and gave a close resemblance to the estimation of the population growths. A steady growth trend was followed by a steeper upward trend that started in most of the age groups at about the end of the 1970s and extended well beyond the 1980s. These results are in agreement with census data published in other developed countries. In Western countries, such as Britain, Sweden and Italy, the growth rates showed an upward trend around the end of the nineteenth century that extended well beyond the Second World War [ Hatton and Martin 2010; Molitoris 2017; Helliwell and Putnam 1995].

In our study, the sex ratio in the Libyan population was skewed towards males. The number of girls per 1000 boys aged $0-9$ years was 991 in 1954, 965 in 1964, and 974 in 1973, and from 960 to 970 between 1984 and 2006, suggesting that fewer girls are born. Similar sex ratios have also been recorded in other developing countries, most notably India, where 959962 girls were born for every 1000 boys in 1981 and 4887
200 I [Lee etal., 20I5]. Further studies are needed to explain the unequal sex ratio in Libyan society. The population profile in Libya has changed substantially during the last fifty years. The proportion of children aged $0-9$ years had increased from $28 \%$ in 1954 to $35 \%$ in 1985 and then declined to $25 \%$ after 1995 . This trend could be interpreted as a shift away from having many children towards providing a better quality of life for children and parents. Similar trends reported in other developing countries, including Latin American and Asian countries; has contributed to the level of urbanization, female education, and the improved life-expectancy of new-born children [ Matus-Lopez and Pedraza 2016; Conde-Agudelo etal., 2012; Lloyd-Sherlock 2014]. Libya has been reported among the top African and Arab countries in education and child health care practice [Daw \& Elkhammas 2008: United nation Report-HDI 201I; Daw etal. 2018]. The age profile has a major contribution to the entity of the Libyan population. Those aged from 10 to 59 years ranged from $55 \%$ to $58 \%$ between 1953 and 1973 to reach 59-67\% between 1994 and 2006. Those aged over 60 years ranged from $5 \%$ in 1954 to $6 \%$ in 2006 . In the first three decades, women comprised about $48 \%$ of the Libyan population and $49 \%$ by 2006 . The workingage population increased by more than ten percentage points due to an increase in the number of women. What is less clear is the effect of these demographic shifts, particularly how they are reflected in the work force, and whether women are represented proportionately across the wide spectrum of occupations. Statistical data continuously reveal that not every qualified individual has an equal opportunity to work in a range of occupational fields. The labor market is not sex neutral and the longstanding occupational disparities between women and men are evident in Arab countries. Thus, this study provides an important analysis of the demographic and employment context within the Libyan population, which could be reflected on demographic changes in the workforce indicated by http://aps.journals.ac.za 
the diversity in age and sex [Cervellati and Sunde $20 \mathrm{II}$. Further studies are needed to understand the relationship between demographic diversity in the general population, development, and choices in the labor market.

Mortality is the most sensitive indicator of the health disparities between poorer and richer nations. It clearly reflects the quality of life and is affected by modernization of health care practices in any country [Aksan and Chakraborty 20I4; Haines 20ll]. The mortality patterns among Libyans remained virtually unchanged between 1954 and 1973. The sustained decline in population mortality in Libya did not begin until the 1970s onwards. Within 50 years, the mortality rate in the country declined by more than two-thirds. This is clearly evident in 2006, where the mortality rate notably declined in all age groups. It ranged between $0.0 \%$ and $0.5 \%$ among those aged between those aged $0-45$ years and was up to $6 \%$ in those aged above 60 years, but there was no sex disparity. This may reflect declines in some of the deadliest diseases of the past and improvement of the socioeconomic status of the country. Tuberculosis, measles and pertussis, for instance, were rampant in Libya till the 1970s. By the 1990s, clean water access had improved for everyone, and electricity had reached most of the population. Moreover, Libya is the only African country where children have easy access to free vaccination and better nutrition [Daw etal. 2016; Daw etal. 2018 ]. Similar results were observed in developed countries during the first quarter of the 20th century. In European countries, deaths from all causes were under 2 deaths per 1,000 for nearly the entire period till 1926 [Post etal. 1997; Szreter 2003]. In this study, the causes of death were not investigated and we were not able to quantify the influence of various factors on overall mortality differentials. Hence, further studies are needed to understand the inequalities of mortality and its causes.

Our study showed substantial variation in the age trends of the Libyan population. Most Libyans are aged $10-59$ years and only $5 \%$ are above 50 years of age, without significant changes in the pattern of death. Good fertility and declining mortality rates have been a major contributing factor to Libya's demographic changes. Similar results were reported in other African and Latin American countries [UN Millennium Report 2005; World Health Report 2006]. We suggest that the proportion of the working age population has been increasing in Libya. This should useful for identifying certain policy directions that could be reflected in greater economic growth in the country. Released censuses usually consist of limited statistics that have been debated by scholars and policy makers, and some people are always missed in every census count, particularly in a large and sparsely populated country like Libya. Places that are undercounted in the census receive little attention and a limited proportion of the available national resources [43, 44 Anderson and Fienberg 200I; O'Hare 2015]. Mortality rates and causes of death are not well documented in decennial census; affordable and sustainable approaches to data collection on mortality that are representative of populations are required. Definitely, complete registration of deaths with full medical certification is the most appropriate means to monitor the health of populations [Alkema etal., 20।6].

\section{Concluding remarks}

This is to be considered the first study in the Arab and North African countries that used national census to analyse the demographic parameters and changes that occurred within the Libyan society over last fifty years. The study provides a first attempt to examine the Libyan population demography, age structure and ability to contribute to the work force. Major sociodemographic factors in Libya are clearly evident from the population's structure. The population trends have shown a low mortality patterns and an increase in fertility rates emerging from the early 1970s.

\section{Recommendations}

The findings of this study could be used to guide strategies to improve the labor sector and strengthen the health care system in the country. Such census biodata could be useful in conducting epidemiologic analyses as diverse as maternal mortality and viral hepatitis and HIV infection rates [Daw etal., 20I4; Austin 2016].

\section{Conflict of interest}

The authors declare that they have no conflict of interests.

\section{References}

African Center for Statistics: Africa Addendum to the United Nations Principles and Recommendations for Population and Housing Censuses, Rev. 2, Draft 2008, dated March 2008.

Aksan, A. \& Chakraborty, S., 20I4. Mortality versus morbidity in the demographic transition.European Economic Review 70(I): 470-492. doi:10.1016/j.euroecorev.2014.06.01I.

Alkema. L., Chou D., Hogan D, Zhang, S., Moller, AB, Gemmill, A, Fat, DM, Boerm,a T, Temmerman, M., Mathers, C., Say, L.,2016 Global, regional, and national levels and trends in maternal mortality between 1990 and 2015 , with scenario-based projections to 2030: a systematic 
analysis by the UN Maternal Mortality Estimation Inter-Agency Group. The Lancet. 2016 Feb

Andersen, O. 1991. "Occupational impacts on mortality declines in the Nordiccountries." In: W. Lutz, (ed.) Future demographic trends in Europe and North America. What can we assume today? London: Academic Press, pp. 4I-54.

Anderson, M. \& Fienberg, S.E. (200I). Who counts? The politics of census taking in contemporary America, New York: Russell Sage Foundation.

Anderson, M. \& Fienberg, S.E. (200I). Who counts? The politics of census taking in contemporary America, New York: Russell Sage Foundation.

Anyangwe. SC., Mtonga C.,2007 Inequities in the global health workforce: the greatest impediment to health in sub-Saharan Africa. International journal of environmental research and public health. Jun 30;4(2):93-100.

Austin, P.C., Lee, D.S., and Fine, JP. 2016. Introduction to the analysis of survival data in the presence of competing risks. Circulation 133(6): 601

609.doi:10.1 I6I/CIRCULATIONAHA.II5.0I77.

Booysen F, Van Der Berg S, Burger R, Von Maltitz M, Du Rand G.,2008. Using an asset index to assess trends in poverty in seven Sub-Saharan African countries. World Development. Jun 30;36(6): I I I 3-30.

Byars-Winston, A., Fouad N., Wen Y, Race/ethnicity and sex in US occupations 2015. 1970-2010: Implications for research, practice, and policy. Journal of vocational behavior. Apr 30;87:54-70

Cai, Y., China's new demographic reality: Learning from the 2010 census. 2013. Population and development review. Sep I;39(3):37I-96.

Cervellati, M., and Sunde,, U.. 20I I. Life expectancy and economic growth: The role of the demographic transition. Journal of Economic Growth 16(2): 99-133. doi:10.1007/s |0887-01।9065-2.

Coast E, Fanghanel A, Lelièvre ,E., Randall, S. 2016. Counting the Population or Describing Society? A Comparison of English and Welsh and French Censuses. European Journal of Population. May I;32(2): 165-88.

Conde-Agudelo, A., Rosas-Bermudez, A., Castaño, F., and Norton, M.H. 2012. Effects of birth spacing on maternal, perinatal, infant, and child health: A systematic review of causal mechanisms. Studies in Family Planning 43(2): 93- II4. doi: I 0. I I I I/j. I 728-4465.20 I2.00308.x.

Daw MA, Ali LA, Daw AM, Sifennasr NE, Dau AA, Agnan MM, El-Bouzedi A. 2018. The geographic variation and spatiotemporal distribution of hepatitis C virus infection in Libya: 2007-2016. BMC infectious diseases. Dec; 18(I):594.
Daw MA, Daw AM, Sifennasr NE, Draha AM, Daw AM, Daw AM, Ahmed MO, Mokhtar ES, ElBouzedi A, Daw IM. 2018. The Epidemiology of Hepatitis D Virus in North Africa: A Systematic Review and Meta-Analysis. The Scientific World Journal. 2018;2018.

Daw MA, Daw AM, Sifennasr NEM, Draha AM, Daw AA, Daw AA, Ahmed MO, Mokhtar ES, ElBouzedi AH, Daw IM, Adam SI, Warrag S. 2019 In association with Libyan Study Group of Hepatitis \& HIV Spatiotemporal analysis and epidemiological characterization of the human immunodeficiency virus (HIV) in Libya within a twenty five year period: 1993-2017. AIDS Res Ther. Jun 25;I6(I):I4. doi: $10.1|86 / \mathrm{s}| 298|-0| 9-0228-0$. PMID: 3| 238947

Daw MA, El-Bouzedi A. 20I4. Prevalence of hepatitis $B$ and hepatitis $C$ infection in Libya: results from a national population based survey. BMC infectious diseases. Jan 9; I4(I): 17.

Daw MA, El-Bouzedi A, Dau AA. 2016. The assessment of efficiency and coordination within the Libyan health care system during the armed conflict-20II. Clinical Epidemiology and Global Health. Sep 30;4(3): I 20-7.

Daw, MA, and. Elkhammas EA. 2008. "Libyan medical education; time to move forward." Libyan Journal of Medicine I-3.

Daw MA, El-Bouzedi AH, Dau AA. 2019. Trends and patterns of deaths, injuries and intentional disabilities within the Libyan armed conflict: 201220I7. PloS one. May 10; 14(5):e02 I606I.

Daw MA, Shabash A, El-Bouzedi A, Dau AA.2014. Seroprevalence of HBV, HCV \& HIV co-infection and risk factors analysis in Tripoli-Libya. PloS one. Jun 17;9(6):e98793.

Day C, Gray A, Budgell E. 20I I. Health and related indicators.South African health review. 201I Jan I;(I): I I 9-248

Faraway JJ. Linear models with R. CRC press; 2014 Jul I.

Groves, R.M. 2010. Director's blog: Quality in a census, some overview thoughts. http://blogs.census.gov/directorsblog/2010/09/qual ity-in-a-census-some-overview-thoughts.html (July 20II).

Haines, M.R. 20I I.Inequality and infant and childhood mortality in the United States in the twentieth century. Explorations in Economic History 48(3): 4I8-428. doi:10.1016/j.eeh.2011.05.009.

Hatton, T.J. 20I I. Infant mortality and the health of survivors: Britain, 19/0-50. Economic History Review 64(3): 95I-972. doi:I0.1 III/j.I4680289.2010. 00572.x.

Hatton TJ, Martin RM. 20I0. Fertility decline and the heights of children in Britain, 1886- 
1938.Explorations in Economic History. Oct 31;47(4):505-19.

Helliwell JF, Putnam RD. 1995.Economic growth and social capital in Italy.Eastern economic journal. Jul I;2I(3):295-307.

Hsu CY, Hu GC. 2014. Beyond Neglect: Long-term Care Research in Low and Middle Income Countries. International Journal of Gerontology. Sep 1;8(3): 107.

Huselid MA.1995. The impact of human resource management practices on turnover, productivity, and corporate financial performance. Academy of management journal. Jun I;38(3):635-72.

Lee J, McGovern ME, Bloom DE, Arokiasamy P, Risbud A, O'Brien J, Kale V, Hu P.20I5. Education, gender, and state-level disparities in the health of older Indians: Evidence from biomarker data. Economics \& Human Biology. Dec 3|; | 9: | 45-56

Lloyd-Sherlock P. 20I4. Beyond neglect: long-term care research in low and middle income countries. International Journal of Gerontology. Jun 30;8(2):66-9.

Lutz, W., Goujon, A., KC, S. and Sanderson, W., 2007. Reconstruction of populations by age, sex and level of educational attainment for 120 countries for 1970-2000. Vienna yearbook of population research, pp. 193-235.

Matus-Lopez M, Pedraza CC. 2016. New Long-Term Care Policies in Latin America: The National System of Care in Uruguay. Journal of the American Medical Directors Association. Jul I; I7(7):663-5

Meyer BD, Mok WK, Sullivan JX. 20I5. Household surveys in crisis. The Journal of Economic Perspectives. Dec I;29(4): 199-226.

Molitoris JJ. 2017. Disparities in death: Inequality in cause-specific infant and child mortality in Stockholm, 1878-1926. Demographic Research. http://www.demographic-

research.org/Volumes/Vol36/I5/

DOI: 10.4054/DemRes.2017.36.15

O'Hare WP. 2015. The undercount of young children in the US Decennial Census. Springer International Publishing; Jun 5. 5;387(I00 I 7):46274.

Post W, Van Poppel F, Van Imhoff E, Kruse E.1997. Reconstructing the extended kin-network in the Netherlands with genealogical data: Methods, problems, and results. Population Studies. Nov I;5I(3):263-78
Ribeiro P, Petyt M. 199.Geometrical non-linear, steady state, forced, periodic vibration of plates, part I: model and convergence studies. Journal of Sound and Vibration. 1999 Oct 7;226(5):955-83.

Turner BL, Hydén G, Kates RW.1993. Population growth and agricultural change in Africa 1993.

Ross NA, Wolfson MC, Dunn JR, Berthelot JM, Kaplan GA, Lynch JW. 2000.Relation between income inequality and mortality in Canada and in the United States: cross sectional assessment using census data and vital statistics. Bmj. Apr I;320(7239):898-902.

Shetty, P., 2012. Grey matter: ageing in developing countries. Lancet 379, I285-1287

Szreter S. 2003. The population health approach in historical perspective. American Journal of Public Health. Mar;93(3):42I-3I

Tejada CA, Triaca LM, da Costa FK, Hellwig F. 2017.The sociodemographic, behavioral, reproductive, and health factors associated with fertility in Brazil.PLoS One. Feb 10; I2(2):e0|7|888. doi: 10.1371/journal.pone.0171888. PMID:28187|67

United Nations. Population Division. World population prospects: the 1994 revision. United Nations; 1995.

United Nation Human Development Index- Libya Country Profile, Posted by Alexandra Valiente on March 5, 201I, https://libyadiary.wordpress.com/201 I/03/05/libya -un-hdi-country-profile/

United Nation Millennium Project, United Nations. Economic Commission for Latin America. The Millennium Development Goals: A Latin American and Caribbean Perspective. United Nations Publications; 2005.

Vogel M, Porter LC.2016. Toward a Demographic Understanding of Incarceration Disparities: Race, Ethnicity, and Age Structure. J Quant Criminol. 20।6;32(4):5 I5-530.PMID: 27928I96

Wang H, Dwyer-Lindgren L, Lofgren KT, Rajaratnam JK, Marcus JR, Levin-Rector A, Levitz CE, Lopez $A D$, Murray CJ. 2013. Age-specific and sexspecific mortality in 187 countries, 1970-20I0: a systematic analysis for the Global Burden of Disease Study 2010. The Lancet. Jan 4;380(9859):207|-94.

World Health Organization Report (WHO) 2006: working together for health. Geneva: World Health Organization, 2006 